\title{
Valsalva Sinus Aneurism Associated with Interventricular Septal Defect, Right Medioventricular Stenosis and Bicuspid Aortic Valve
}

\author{
Momar Sokhna Diop*, Papa Salmane Ba, Papa Amath Diagne, Papa Ousmane Ba, \\ Amadou Gabriel Ciss \\ Department of Thoracic and Cardiovascular Surgery, Faculty of Medicine, Cheikh Anta Diop University, Dakar, Senegal \\ Email address: \\ momarsokhna08@yahoo.fr (M. S. Diop) \\ ${ }^{*}$ Corresponding author

\section{To cite this article:} \\ Momar Sokhna Diop, Papa Salmane Ba, Papa Amath Diagne, Papa Ousmane Ba, Amadou Gabriel Ciss. Valsalva Sinus Aneurism \\ Associated with Interventricular Septal Defect, Right Medioventricular Stenosis and Bicuspid Aortic Valve. International Journal of \\ Cardiovascular and Thoracic Surgery. Vol. 7, No. 4, 2021, pp. 46-49. doi: 10.11648/j.ijcts.20210704.11
}

Received: August 24, 2021; Accepted: September 16, 2021; Published: September 30, 2021

\begin{abstract}
Backgroud: Valsalva sinus aneurysms are abnormalities of the heart that can be acquired or congenital. The most often associated congenital anomaly is interventricular communication which is a predisposing factor to the occurrence of aortic insufficiency. Transcatheter closure has recently emerged as an alternative but surgery still has indications. Aim: We report our experience on the management of this pathology in a subsaharian country. Patient and method: We report the observation of a 7.5-year-old child whose discovery was fortuitous upon exploration of a heart murmur. Results: Doppler echocardiography made the diagnosis possible showing a large under pulmonary interventricular septal defect with a right left shunt, a small non-stenotic sub-aortic membrane, a bicuspid aortic valve without leakage or stenosis, a significant dilation of the aortic root. The surgical management was an intervention according to Tyrone-David technique with closure of the interventricular septal defect. Postoperative outcomes were simple and the short- to medium-term evolution favorable. Conclusion: Depending on the definition used to diagnose valsalva sinus aneurysms, the frequency of this pathology may be underestimated. What seems most appropriate to us is the definition considering the diameter of the sinuses relative to the body surface. An another endpoint is the need to explore the aortic root in the presence of interventricular communication and bicuspid aortic valve.
\end{abstract}

Keywords: Valsalva Sinus Aneurysm, Interventricular Septal Defect, Surgery

\section{Introduction}

Valsalva sinus aneurysms are heart abnormalities that can be acquired or congenital [1]. In most cases, they are due to connective tissue abnormalities, infections, trauma or degenerative diseases. These aneurysms are often asymptomatic and are diagnosed incidentally or when ruptured in nearby structures or when performing imaging tests for other indications [2]. The most often associated congenital anomaly is interventricular communication which is a predisposing factor to the occurrence of aortic insufficiency due to the venturi effect, which often determines the prognosis [3]. Transcatheter closure has recently emerged as an alternative, less invasive treatment for patient population who are isolated sinus of valsalva aneurysm [4].

\section{Observation}

We report the observation of a child aged 7.5 years received in our department for the surgical management of an interventricular septal defect associated with a valsalva sinus aneurysm discovered in context of an asthmatic dyspnea with hyperactivity in the precordium. He is 2 nd in a family of 4 children, one of whom died in the neonatal period of unknown cause. He was the result of a full-term pregnancy with eutocic vaginal delivery without any notion of resuscitation at birth. 
There was no notion of family consanguinity. On physical examination, he presented in good general condition with normostained mucous membranes. There were no peripheral signs of heart congestion. Her admission constants were $18 \mathrm{kgs}$ weight, $115 \mathrm{~cm}$ height, $113 \mathrm{mmHg}$ systolic and $83 \mathrm{mmHg}$ diastolic blood pressure, 97 beats per minute heart rate, $99 \%$ pulsed oxygen saturation, temperature at $37^{\circ}$ celcius. On cardiac auscultation, heart sounds were heard at all foci with a mesocardiac systolic murmur of 4 / 6th intensity and a burst of B2 at the pulmonary focus. The electrocardiogram showed a regular sinus rhythm with a frequency of 107 cycles per minute, incomplete right bundle branch block, left atrial enlargement. On the chest x-ray, cardiomegaly was noted with a cardiothoracic index of 0.58 . The echodoppler cardiac showed a large under pulmonary interventricular septal defect with a right left shunt, a small non-stenotic sub-aortic membrane, a bicuspid aortic valve without leakage or stenosis, a significant dilation of the aortic root to $35 \mathrm{~mm}$ ( $\mathrm{z}$ score at +9 ) with an aortic sinus measured at $33 \mathrm{~mm}(\mathrm{z}$ score at +8$)$, a medioventricular stenosis with a maximum pulmonary gradient at $83 \mathrm{mmhg}$. The pulmonary trunk and branches were correct with a patent foramen ovale shunting left to right. There was the presence of a small $2.5 \mathrm{~mm}$ ductus arteriosus. He underwent a closure of the interventricular septal defect by a heterologous patch with medioventricular muscle resection, a closure of the permeable foramen ovale, a replacement of the ascending aorta by a $22 \mathrm{~mm}$ Dacron tube according to the Tyrone-David technique and a ligation of the ductus arteriosus. Intraoperative transesophageal echocardiography showed good biventricular function with no residual shunt. The maximum gradient over the aorta was $8 \mathrm{mmHg}$. The immediate postoperative period was marked by hypertensive outbreaks requiring her to be placed on nicardipine and captopril with a good clinical outcome. Extubation was performed seven hours after operation. The weaning of the amines (noradrenaline and dopamine) was carried out at three hours postoperativily and the corotrope at the second day after operation. Postoperative control transthoracic echocardiography showed the aortic tube measured at $22 \mathrm{~mm}$, no valvular aortic insufficiency, moderate right ventricular hypertrophy, a pulmonary residual gradient at $15 \mathrm{~mm} \mathrm{Hg}$. Drain ablation was performed at the second day postoperativily. The length of stay in intensive care unit was 48 hours. While in hospital, he developed an infectious syndrome with isolation from the blood culture of a staphylococcus aureus susceptible to chloramphenicol, ciprofloxacin and erythromycin. A good evolution was noted with apyrexia and a regression of the biological inflammatory syndrome under directed antibiotic therapy. The length of hospitalization was 15 days. The exit treatment was ciprofloxacin, captopril, aspegic and lasilix. One month later, an echocardiography found a tiny aortic insufficiency, the maximum gradient over the aortic valve was $9 \mathrm{mmHg}$. At the last echocardiography control (two years later), we found a bicuspid aortic valve with a tiny leak without stenosis (maximal aortic valve gradient at $10 \mathrm{mmHg}$ ), a medioventricular maximal gradient at $13 \mathrm{mmHg}$.

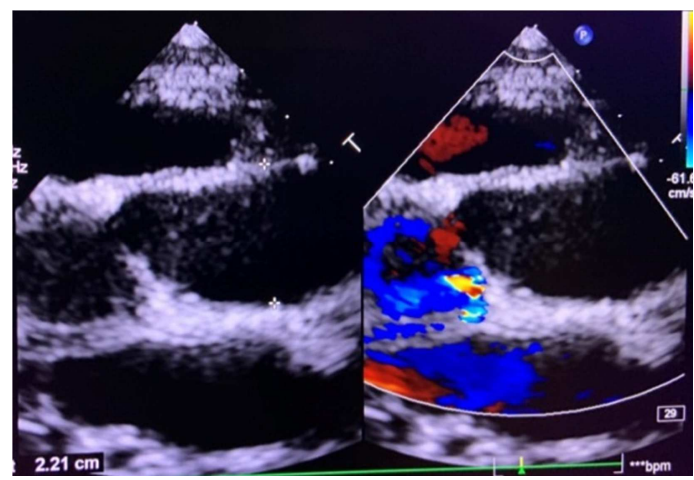

Figure 1. Postoperative Echocardiography shows the left ventricular outflow tract.

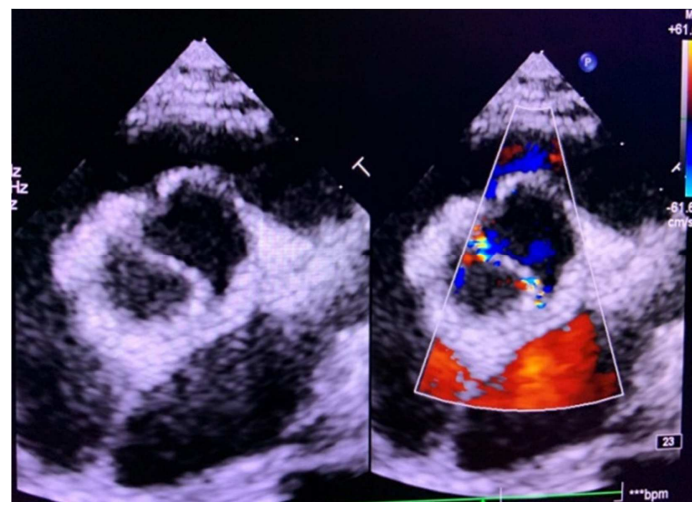

Figure 2. Postoperative echocardiography shows the bicuspid aortic valve.

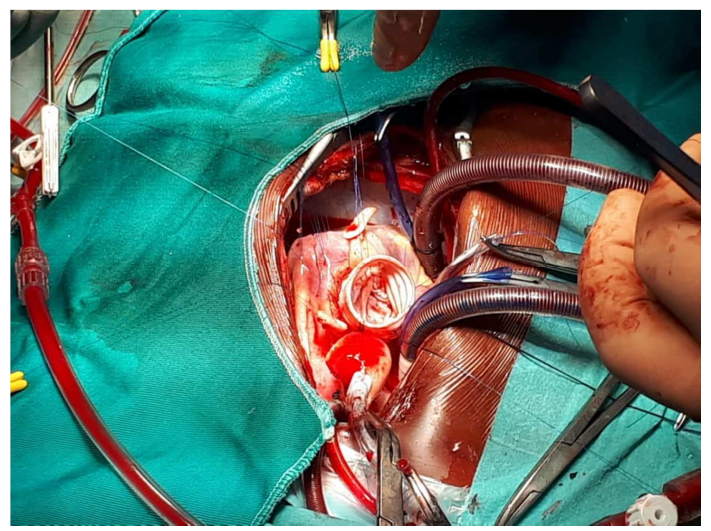

Figure 3. Operative view shows aortic dacron tube.

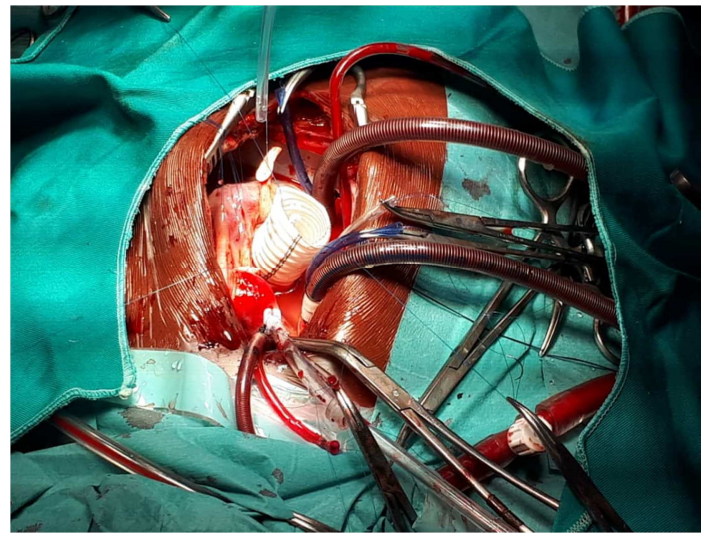

Figure 4. Operative view shows aortic dacron tube and the right coronary artery before reimplantation. 


\section{Discussion}

Aortic root aneurysm is commonly found in patients with congenital heart disease. This dilation is correlated with a risk of aortic insufficiency or rupture. The most common congenital heart disease is ventricular communication [5]. Sinus of valsalva aneurysm most often originate from right or non-coronary sinus and only rarely from the left coronary sinus. These lesions usually remain silent and undetected unless rupture occurs. Importantly, left ventricular ejection fraction was the only baseline imaging parameter that was independently associated with increased probability of sinus of Valsalva aneurysm rupture at presentation. A history of statin use was significantly associated with reduced likelihood of sinus of Valsalva aneurysms rupture at presentation [6]. The impact of statin treatment on ascending aorta aneurysm was evaluated in a propensity score-matched case-control study [7]. However, there are currently no data on the efficacy of statin treatment in sinus of Valsalva aneurysms [6]. Unruptured sinus of valsalva aneurysm can cause important structural and electrophysiologic, abnormalities that turn lead to arrhythmia, heart block, myocardial ischemia and rarely right ventricular outflow tract obstruction [8]. In the presented case, it was after an exploration of the interventricular septal defect that the valsalva sinus aneurysm was diagnosed. They described a theory how preexisting ventricular septal defect below the location of the sinus of Valsalva may contribute to the development of aneurysm formation and eventually lead to rupture [9]. Although sinus of Valsalva aneurysms are reported to have a higher prevalence in Asian and Far eastern population [10]. Echocardiography and multimodality imaging in the contemporary era play key roles in a diagnosis and management of sinus of Valsalva aneurysm. Transthoracic echocardiography is considered the first-line imaging modality for its convenience and excellent diagnostic accuracy [11]. However, studies have shown commonly associated pathologies such as ventricular septal defect, right ventricular outflow tract stenosis, and aortic valve insufficiency can cause diagnostic challenges for transthoracic echocardiography [12]. Transesophageal echocardiography on the other hand, provides superior imaging quality [9]. We speak of an aneurysm if the ratio between the diameter of the valsalva sinus and the body surface area is greater than $2.1 \mathrm{~cm} / \mathrm{m}^{2}$ or if the diameter of the valsalva sinus in absolute value $\geq 4.0 \mathrm{~cm} \mathrm{[2].} \mathrm{In} \mathrm{our}$ patient, this diameter was $3.3 \mathrm{~cm}$ in absolute value, therefore less than $4 \mathrm{~cm}$, on the other hand the ratio between the diameter of the valsalva sinus and the body surface area was $4.4 \mathrm{~cm} / \mathrm{m}^{2}\left(>2.1 \mathrm{~cm} / \mathrm{m}^{2}\right)$. This discrepancy between definitions can caused by inclusion of body size in aortic root dilatation (ARD) definition. Absolute aortic diameter (Valsalva $\geq 4 \mathrm{~cm}$ ) as cutoff value for ARD is simple and clinically feasible; however, this definition underestimated the prevalence of ARD compared to the definitions of ARD based on observed aortic root diameter/ body surface area
$>2,1 \mathrm{~cm} / \mathrm{m}^{2}$ [5]. Management of unruptured sinus of Valsalva aneurysm remains controversial, partly because dilatation of aorta at this point (in the absence of underlying connective tissue disease) seems have a less malignant prognosis than does dilatation of other of aorta. However, current guidelines advise surgical correction if the diameter of sinuses exceed $5,5 \mathrm{~cm}$ or if significant valvular dysfunction is noted [8]. In our patient, the indication was given because of the associated lesions, namely the interventricular septal defect, the right medioventricular stenosis which required surgical treatment under extracorporeal circulation and because of the sinus diameter / body surface ratio was greater than $2.1 \mathrm{~cm} / \mathrm{m}^{2}$. The other important fact was the observation of valsalva sinus aneurysms noted during the follow-up of patients who have benefited from isolated interventricular septal defect closure [5]. The third decision factor is association with bicuspid aortic valve. About half of young patients with bicuspid aortic valve show evidence of aortic dilatation on echocardiography, which increases the risk of dissection by a factor of 9 [13]. Each of these malformations (sinus of Valsalva aneurysm, perimembranous ventricular septal defect and bicuspid aortic valve) could explain the development of progressive aortic valve regurgitation. At the same time, there always existed the risk of a gradual or abrupt progression of a small perforation of the coronary sinus aneurysm. Considering various risks, when aortic valve regurgitation with cusp prolapse appeared before the progression of coronary sinus rupture, surgical closure of the ventricular septal defect and repair of the aortic sinus aneurysm even in the absence of symptoms was considered carefully [14]. Our patient underwent an ascending aorta replacement using the Tyrone-David technique with closure of septal defects and right mid-ventricular muscle resection. The postoperative prognosis seems better if there is preservation of the native aortic valve can avoid subsequent prosthetic dysfunction [15]. Aortic valve function should be assessed carefully preoperatively, and during operation the need for valve reconstruction or replacement should be considered comprehensively and in conjunction with the aortic root and adjacent structure [3].

\section{Conclusion}

This observation shows the need to explore the aortic root in the presence of interventricular communication and bicuspid aortic valve. In addition, depending on the definition used to diagnose valsalva sinus aneurysms, the frequency of this pathology may be underestimated. What seems most appropriate to us is the definition considering the diameter of the sinuses relative to the body surface. The indication for surgery in this specific case is based on a bundle of arguments, all the more so that the $5.5 \mathrm{~cm}$ cut off found in the recommendations was not achieved in this patient. In children, great care should be taken in the surgical treatment to conserve the aortic valve to improve the long-term prognosis. 


\section{References}

[1] Goldberg N., Krasnow N. Sinus of Valsalva aneurysms. Clin. Cardiol. 13, 831-836 (1990).

[2] Soichiro Henmi, Koki Yokawa, Yutaka Okita. Right ventricular outflow tract obstruction caused by sinus of Valsalva aneurysm. General Thoracic and Cardiovascular Surgery https://doi.org/10.1007/s11748-020-01546-5.

[3] Khakimjon Abralov, Amonjon Alimov. Short term results of sinus of Valsalva aneurysm repair. World Journal for Pediatric and Congenital Heart Surgery. 2017, Vol. 8 (1) 13-17.

[4] Liang Tang, Sheng-Hua Zhou, Zhen-Fei Fang. Transcatheter closure of ruptured sinus of Valsalva aneurysm with Doubledisc perimembranous VSD occlude in man with mechanical aortic valve. Tex Heart Inst J 2019; 46 (3): 211-4.

[5] Saito C, Fukushima N, Fukushima K, Matsumura G, Ashihara K, Hagiwara N. Factors associated with aortic root dilatation after surgically repaired ventricular septal defect. Echocardiography. 2017; 00: 1-7.

[6] Bo Xu, Doygu Kocyigit, Carlos Godoy Rivas and al. Outcomes of contemporary imaging-guided management of sinus of Valsalva aneurysms. Cardiovasc Diagn Ther 2021; 11 (3): $770-780$.

[7] Angeloni E, Vitaterna A, Pirelli M, et al. Effects of statin therapy on ascending aorta aneurysm growth: A propensitymatched analysis. Int J Cardiol 2015; 191: 52-5.

[8] Sambhunath Das, Suruchi Ladha, and Balram Airan. Unruptured sinus of Valsalva aneurysm with RVOT obstruction and supracristal ventricular septal defect-A rare combination. Echocardiography 2015; 32: 1322-1324.
[9] Kei Togashi, Francisco J, Gensini Paez, Richard Shen. Sinus of Valsalva aneurysm rupture associated with a ventricular septal defect: the importance of multi-Angle assessment by intraoperative transoesophageal echocardiography. Journal of Cardiothoracic and vascular anesthesia 000 (2020) 1-6 Article in Press.

[10] Wang Z. J, Zou C. W, Li D. C and al. Surgical repair of sinus of Valsalva aneurysm in Asian patients. Ann Thorac Surg 2007; 84: 156-60.

[11] Bo Xu, Kocyigit D, Betancor J and al. Sinus of Valsalva aneurysms: A state-of the Art Imaging Review. J Am Soc Echo cardiogr 2020; 33: 295-312.

[12] Cheng T. O, Yang Y. L, Xie M. X et al. Echocardiographic diagnosis of sinus of Valsalva aneurysm: A 17-year (19952012) experience of 212 surgically treated patients from one single medical center in China. Int J Cardiol 2014; 173: 33-9.

[13] Violeta V. Groudeva Borislav G. Atzev Dimitar G. Petkov. Management of late presenting congenital combined heart defect-bicuspid aortic valve and ventricular septal aneurysm. Am J Case Rep, 2020; 21: e919766 DOI: 10.12659/AJCR.919766.

[14] Seung Hyun Lee, Jae Bum Kim, Nam Hee Park, Hyung Seop Kim, Dong Yoon Keum. Asymptomatic Ruptured sinus of Valsalva aneurysm combined with perimembranous ventricular septal defect, and bicuspid aortic valve in adult patient. Thoracic Cardiovasc Surg 2013; 61: 327-329.

[15] Pouya Nezafati, Mohammad Hassan Nezafati, and Hamid Hoseinikhah. Unruptured aneurysm of sinus of Valsalva coexisting with the large ventricular septal defect and severe aortic regurgitation in a young man. Case Reports in Medicine. Volume 2015, Article ID 396098, 3 pages http://dx.doi.org/10.1155/2015/396098. 